\title{
ATP-bound Conformation of OBP Chaperonin
}

\author{
T.B. Stanishneva-Konovalova ${ }^{1}$, E.B. Pichkur ${ }^{2}$, P.I. Semenyuk ${ }^{3}$, L.P. Kurochkina ${ }^{3}$ and O.S. Sokolova ${ }^{*}$ \\ 1. Lomonosov Moscow State University, Faculty of Biology, Moscow, Russia. \\ 2. National Research Center "Kurchatov Institute", Moscow, Russia. \\ 3. Lomonosov Moscow State University, A.N. Belozersky Institute of Physico-Chemical Biology, \\ Moscow, Russia. \\ * Corresponding author: sokolova@mail.bio.msu.ru
}

To be functionally active, all newly synthesized proteins must fold to their native 3D structures. While small proteins can fold spontaneously, larger ones require the assistance of special cell agents called molecular chaperones. The hsp60 class of chaperones, called chaperonins, provide an inner chamber for protein folding. Typically, they form barrel-shaped structures out of two rings stacked back to back, where each ring consists of several subunits and each subunit is comprised of equatorial, intermediate and apical domains. Importantly, the cycle of protein folding inside the chaperonin cage is controlled by ATP binding and hydrolysis, which occurs in the equatorial domain and triggers movements of intermediate and apical domains. Chaperonins are present in all domains of life and are divided into two groups: group I contains bacterial variants, among which GroEL is the most well studied representative, and also mitochondrial and chloroplast variants; group II is present in eukaryotic cytosol and in archaea. However, recently discovered viral chaperonins are not included in this division and possess similarities to both groups, as well as their unique features. Using cryo-EM we previously solved the 3D structure of a chaperonin encoded by the genome of the bacteriophage OBP Pseudomonas fluorescens. Here, we solve its structure in complex with ATP.

The recombinant OBP chaperonin was expressed in E.coli cells and purified using anion-exchange chromatography on Q-sepharose. The purified protein was incubated with $3 \mathrm{mM}$ ATP for 13 min and vitrified in Vitrobot Mark IV (FEI). A total of 4200 images were collected in automated data acquisition mode in Cs-corrected cryo-TEM Titan Krios (FEI) equipped with XFEG-electron source and direct electron detector Falcon II. Particle picking was performed in Warp [1] and then CryoSPARC [2] was used for 2D classification, ab-initio model generation and 3D classification. $\sim 280000$ particles were used for non-uniform refinement in CryoSPARC.

The previously solved nucleotide-free state was a single-ring structure showing a surprising degree of asymmetry despite being assembled from 7 identical subunits [3]. They were arranged in pairs with apicalapical and intermediate-apical salt bridges between subunits within pairs, and only equatorial domains displayed C7 symmetry. The unpaired subunit was more flexible above the equatorial domain, presumably due to the lack of stabilizing contacts, and this subunit was averaged out in the final 3D model. The ATPbound state presented here shows higher conformational variability, as 3 subunits were averaged out in the 3D model. Similar to the nucleotide-free state, local resolution varies significantly from one subunit to another and from the equatorial to the apical domains. Side chains are clearly visible in the alpha helixes in the equatorial domains, which is consistent with the local resolution estimated to be higher than $3.5 \AA$ in this region. Apical domains show high heterogeneity, resulting in the secondary structure been resolved poorly or not resolved at all, which is also supported by the local resolution estimation of 7-12 A. To interpret the structure, we build a homology-based model using the GroEL structure (accession code 1aon in the Protein Data Bank) as a reference and fitted the atomic model into the experimental map with COOT 
[4] (Fig. 1A). An extra density, corresponding to the ATP molecule, was present in the equatorial domains of all subunits (Fig. 1B). Although GroEL is thought to be the ancestor of OBP chaperonin [5], the effects of ATP binding appear to be quite different. In GroEL, ATP binding triggers the rotation of the apical domains, which is enabled by the presence of a "hinge" between the intermediate and apical domains: residues Gly192 and Gly375. In OBP chaperonin, these residues are replaced by large polar residues Glu191 and Asn376, which, unlike glycine residues, cannot provide cis/trans switching. As a result, the upper domains' rotation was not observed. Thus, OBP chaperonin may have developed a different protein folding cycle [6].

\section{References:}

[1] D Tegunov and P Cramer, bioRxiv, 338558 (2018).

[2] A Punjanji et al., Nat Methods 14 (2017), p. 290.

[3] TB Stanishneva-Konovalova et al., Microsc. Microanal. 24(S1) (2018), p. 1244.

[4] P Emsley and K Cowtan, Acta Crystallogr. Sect D 60 (2004), p. 2126.

[5] PI Semenyuk et al., Biochem. J. 473 (2016), p. 2383.

[6] The authors acknowledge funding from the Russian Foundation for Basic Research (Grant \#19-0400605 to O.S.S., \#18-04-01281 to L.P.K.). E.B.P. was supported by RSF grant \#18-41-06001. The cryoEM data collection has been performed at the Resource Center for Probe and Electron Microscopy at the NRC "Kurchatov Institute".
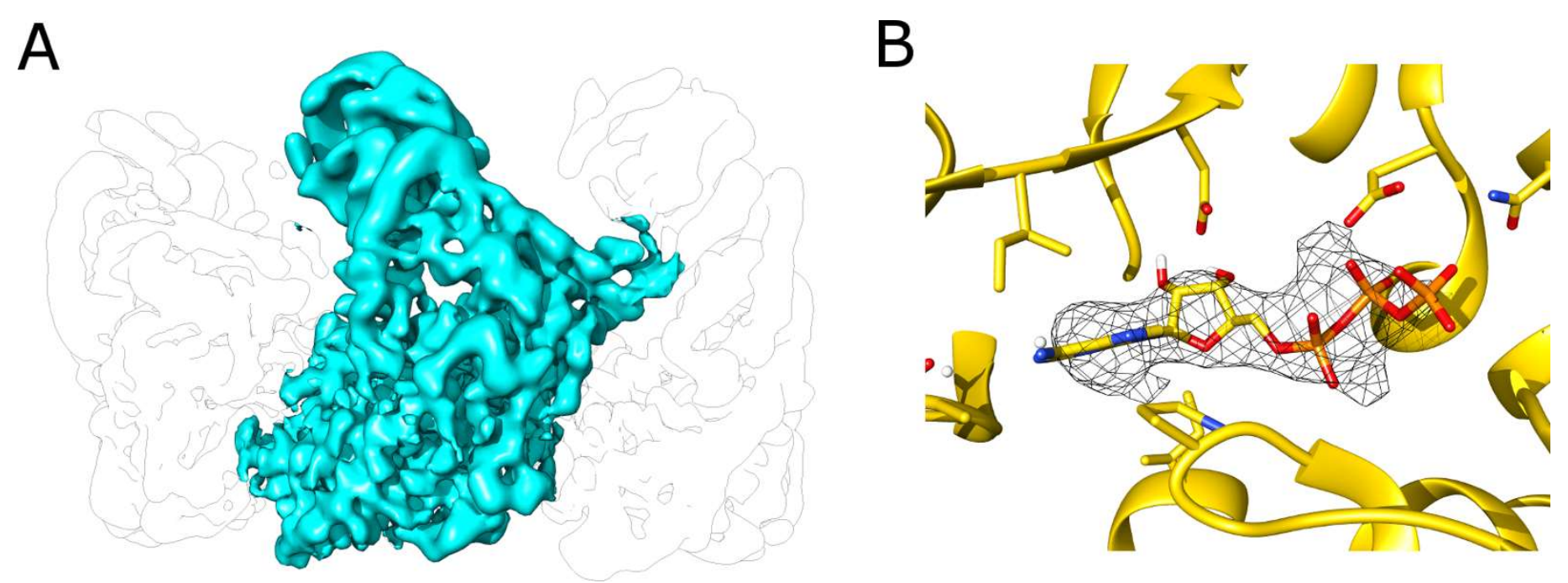

Figure 1. (A) Cryo-EM structure of the ATP-bound state of the OBP chaperonin with one subunit colored blue; (B) Density in the ATP-binding pocket of the OBP chaperonin (mesh) with an atomic model of an ATP-bound GroEL. 